\title{
Progress in microwave to sub-THz sources at Strathclyde
}

\author{
A. D. R. Phelps \\ Physics Department, SUPA, University of Strathclyde, Glasgow G4 0NG, Scotland, UK \\ a.d.r.phelps@strath.ac.uk
}

Research progress during recent years at the University of Strathclyde is presented. The main research themes include gyro-devices with particular emphasis on gyro-TWAs [1,2] and gyro-BWOs, and natural sources of radiation such as auroral kilometric radiation (AKR) [3-6], novel Cherenkov mm-wave sources [7,8] and pseudospark-driven sub-THz sources [9-11].

While there has been some theoretical work, the majority of the research has involved either laboratory experiments, or numerical simulations.

\section{Gyro-devices}

Most of the work on gyro-devices has involved helical waveguide interaction structures [1,2]. This interest began in the 1990's stimulated by collaborative research with IAP. The early experiments were in the $10 \mathrm{GHz}$ frequency range, whereas our more recent experiments have been in the 90 to $100 \mathrm{GHz}$ range. The applications for these mm-wave amplifiers include DNP-NMR, cloud radar and communications, etc.

\begin{tabular}{|l|c|}
\hline \multicolumn{2}{|c|}{ Gyro-TWA design target } \\
\hline Frequency band & W-band \\
\hline Centre frequency & $95 \mathrm{GHz}$ \\
\hline Bandwidth & $90-100 \mathrm{GHz}$ \\
\hline Power & $\sim 5 \mathrm{~kW}$ \\
\hline Gain & $\sim 40 \mathrm{~dB}$ \\
\hline Efficiency & $10 \%$ \\
\hline $\begin{array}{l}\text { Microwave } \\
\text { output }\end{array}$ & $\begin{array}{c}\text { Fundamental } \\
\text { Gaussian mode }\end{array}$ \\
\hline
\end{tabular}

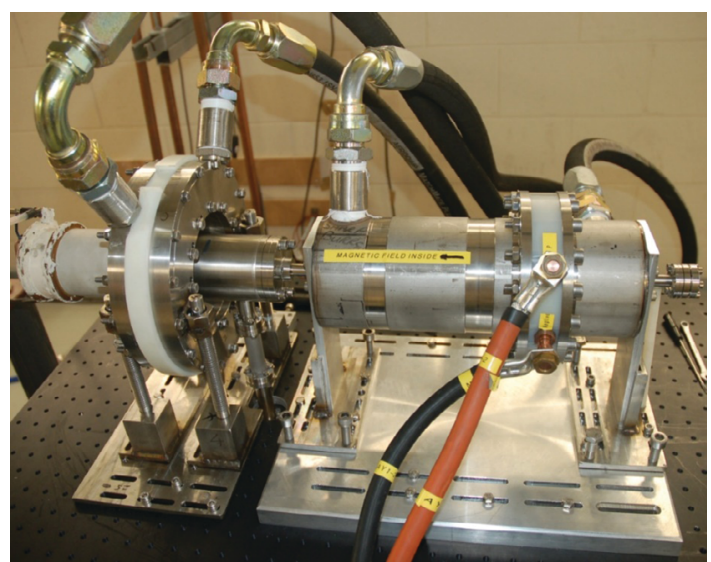

Fig. 1. Gyro-TWA, $90-100 \mathrm{GHz}$

The gyro-TWA shown in Fig. 1 has produced 3.4kW output power. The limit on the output power is presently the maximum power that is available at the input to the amplifier.

\section{Natural sources of EM radiation}

Previous research on laboratory gyro-devices and electron cyclotron masers has provided the background for investigations of the mechanism of some naturally occurring sources of electromagnetic radiation. A research programme stretching over more than 10 years has explored the detailed mechanism of auroral kilometric radiation (AKR) and similar emissions emanating from other astrophysical bodies. Laboratory experiments to simulate the natural phenomena, combined with theoretical analysis and numerical modelling have been compared with natural observations. Using all four approaches it has been possible to understand how AKR arises and the mechanism by which the energy in electron streams can be converted with $1 \%$ or $2 \%$ efficiency into AKR. The mechanism depends upon the existence of an anisotropy in velocity space. This anisotropy is created when electrons are spiralling down the magnetospheric field lines. The magnetic moment is an adiabatic invariant of the electron motion and as the electrons penetrate further into the increasing magnetic field the electrons increase the perpendicular component of their velocity and decrease the component of their velocity parallel to the magnetic field lines. This results in a horseshoe shaped particle distribution in velocity space. The positive value of the gradient with respect to the perpendicular velocity component of the distribution function drives this kinetic instability that is now known as the "horseshoe instability". The theoretical analysis, the PiC simulations, the laboratory experiment and the space observations all agree.

\section{Cherenkov sources}

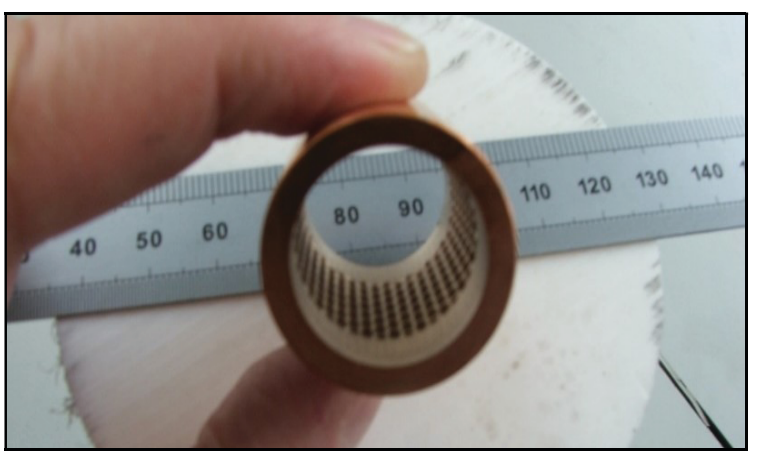

Fig. 2. Cylindrical over-sized Cherenkov structure

One of the aims of the research on Cherenkov sources is to retain reasonably high output power levels as the frequency increases. Traditionally Cherenkov sources have followed the trend that as the frequency increases and the wavelength decreases the interaction structures shrink to retain low order mode 
operation and the power handling capacity decreases as a result. Methods of selecting and driving modes in highly overmoded structures have been explored. Coupling of modes to form an unique eigenvalue is one of the methods that has been shown to be promising to achieve efficient excitation of a single high power mode in an overmoded structure.

Ginzburg et al. [12] have successfully demonstrated the coherent summation of Cherenkov sources, which provides a pathway to achieving very high intensity fields by combining several sources.

\section{Pseudospark-driven sub-THz sources}

Pseudosparks have been known since at least the 1970's and have been developed for several applications including switching. Our interest in pseudosparks is related to their ability to emit intense high brightness electron beams. It has been found that by optimizing the pseudospark geometrical configuration and by employing micro collimating apertures, micro electron beams can be produced that are ideal for driving small structures suitable for creating sub- $\mathrm{THz}$ sources.

A W-band (75-110 GHz) Extended Interaction Oscillator (EIO) millimetre wave source was designed and constructed to operate in the $2 \pi$ mode. With a 35 $\mathrm{kV}$ discharge voltage, the EIO successfully produced $\mathrm{W}$-band radiation pulses of $200 \mathrm{~W}$ peak power and 20ns pulse duration, agreeing with 3D Particle-in-Cell PiC simulations. Simulations show the capability to extend this concept to higher frequencies.

\section{Acknowledgements}

The UK Engineering and Physical Research Council (EPSRC) is thanked for supporting this research.

\section{References}

1. Denisov, G. G., Bratman, V. L., Cross, A. W., He, W., Phelps, A. D. R., Ronald, K., Samsonov, S. V., and Whyte, $C$. G. Gyrotron traveling wave amplifier with a helical interaction waveguide // Phys. Rev. Lett. 1998. V. 81, No. 25, P. 5680-5683.

2. Bratman, V. L., Denisov, G. G., Samsonov, S. V., Cross, A. W., Phelps, A. D. R., and Xe, W. Hgh-efficiency wideband gyro-TWTs and gyro-BWOs with helically corrugated waveguides// Radiophysics and Quantum Electronics. 2007. V. 50. No. 2. P. 95-107.
3. McConville, S. L., Speirs, D. C., Ronald, K., Phelps, A. D. R., Cross, A. W., Bingham, R., Robertson, C. W., Whyte, C. G., He, W., Gillespie, K. M., Vorgul, I., Cairns, R. $A$., and Kellett, B. J. Demonstration of auroral radio emission mechanisms by laboratory experiment // Plasma Phys. Control. Fusion, 2008. V. 50. No. 7. 074010. P.1-13.

4. Speirs, D. C., Vorgul, I., Ronald, K., Bingham, R., Cairns, R. A., Phelps, A. D. R., Kellett, B. J., Cross, A. W., Whyte, C. G., and Robertson, C. A laboratory experiment to investigate auroral kilometric radiation emission mechanisms // J. Plasma Phys. 2005. V. 71. No. 5. P. 665-674.

5. Speirs, D. C., McConville, S. L., Gillespie, K. M., Ronald, K., Phelps, A. D. R., Cross, A. W., Bingham, R., Robertson, C. W., Whyte, C. G., Vorgul, I., Cairns, R. A., and Kellett, B. J. Numerical simulation of auroral cyclotron maser processes // Plasma Phys. Control. Fusion. 2008. V. 50. No. 7. 074011. P. 1-15.

6. Speirs, D. C., Bingham, R., Cairns, R. A., Vorgul, I., Kellett, B. J., Phelps, A. D. R., and Ronald, K. Backward Wave Cyclotron-Maser Emission in the Auroral Magnetosphere // Phys. Rev. Lett.. 2014. V. 113. No. 15. 155002. P. $1-5$.

7. Konoplev, I. V., Fisher, L., Cross, A. W., Phelps, A. $D$. R., Ronald, K., and Robertson, C. W. Surface wave Cherenkov maser based on a periodic lattice // Appl. Phys. Lett 2010. V. 96. No. 26. 261101. P.1-3.

8. Konoplev, I. V., MacLachlan, A. J., Robertson, C. W., Cross, A. W., and Phelps, A. D. R. Cylindrical periodic surface lattice as a metadielectric: Concept of a surface-field Cherenkov source of coherent radiation // Phys. Rev. A. 2011. V. 84. No. 1. 013826. P. 1-12.

9. Yin, H., Robb, G. R. M., He, W., Phelps, A. D. R., Cross, $A$. W., and Ronald, $K$. Pseudospark-based electron beam and Cherenkov maser experiments // Phys. Plasmas. 2000. V. 7. No. 12. P. 5195-5205.

10. Yin, H., Cross, A. W., Phelps, A. D. R., Zhu, D., He, $W$., and Ronald, $K$. Propagation and post-acceleration of a pseudospark-sourced electron beam // J. Appl. Phys. 2002. V. 91. No. 8. P. 5419-5422.

11. He, W., Zhang, L., Bowes, D., Yin, H., Ronald, K., Phelps, A. D. R., and Cross, A. W. Generation of broadband terahertz radiation using a backward wave oscillator and pseudospark-sourced electron beam // Appl. Phys. Lett. 2015. V. 107. No. 13. 133501. P.1-4.

12. Ginzburg, N. S., Cross, A. W., Golovanov, A. A., Mesyats, G. A., Pedos, M. S., Phelps, A. D. R., Romanchenko, I. V., Rostov, V. V., Rukin, S. N., Sharypov, K. A., Shpak, V. G., Shunailov, S. A., Ulmaskulov, M. R., Yalandin, M. I., and Zotova, I. V. Generation of Electromagnetic Fields of Extremely High Intensity by Coherent Summation of Cherenkov Superradiance Pulses // Phys. Rev. Lett.. 2015. V. 115. No. 11. 114802. P. 1-5. 